\title{
Synthesis and Characterization of High Dielectric Constant Material $\mathrm{CaCu}_{3} \mathrm{Ti}_{4} \mathrm{O}_{12}$
}

\author{
Anshuman Srivastava ${ }^{1}, 0 m$ Parkash $^{2}$, Devendra Kumar ${ }^{3}$ and Pralay Maiti ${ }^{4}$ \\ ${ }^{1}$ Department of Mechanical Engineering, SIET Allahabad, India \\ ${ }^{2,3}$ Department of Ceramic Engineering, Indian Institute of Technology, India \\ ${ }^{4}$ School of Material Science \& Technology, Indian Institute of Technology, India
}

Received: September 06, 2017; Published: September 18, 2017

*Corresponding author: Anshuman Srivastava, Department of Mechanical Engineering, SIET Allahabad, India; Email: anshuman0203@gmail.com

\begin{abstract}
Today a lot of advancement is taking place in electronics industry. This advancement is concomitant with the creation of new materials with high permittivity (called high-k dielectric materials). A higher $\mathrm{k}$ dielectric material can store more electric energy than the one with a lower value. As a result, its use in electronic devices reduces the size of the components and improves their efficiency also. As Capacitor is one of the most important components of electronic devices, so lot of research is going on to reduce its size. Traditional $\mathrm{BaTiO}_{3}$ based ferroelectric materials exhibit high dielectric constant. There is a problem with these materials as these have strong temperature dependence of their dielectric constants near ferro to para electric transition. Therefore, $\mathrm{CaCu}_{3} \mathrm{Ti}_{4} \mathrm{O}_{12}$ (CCTO) has attracted increasing scientific and technological interest because of its giant dielectric constant $\left(\mathrm{gr} \sim 10^{4}\right)$ with weak temperature/frequency dependence in a wide range of temperature $(100-600 \mathrm{~K})$ and frequency. This suggests its potential application in capacitor based devices. In the present work, Solid state conventional technique was adopted for the synthesis of $\mathrm{CaCu}_{3} \mathrm{Ti}_{4} \mathrm{O}_{12}$ (CCTO), using $\mathrm{CuO}, \mathrm{TiO}_{2}$, and $\mathrm{CaCO}_{3}$ as starting materials. X-ray diffraction patterns were recorded in an X-ray diffractometer employing $\mathrm{Cu}-\mathrm{K}_{\alpha}$ radiation with a Ni-filter. SEM was recorded to analyze the microstructure. Dielectric measurement has been performed using four probe novocontrol set up (ZG4) in a wide range of temperature. It was observed that dielectric constant increases with the increase in temperature and decreases with the increase in frequency. Dielectric loss was found to be quite low.
\end{abstract}

Keywords: $\mathrm{CaCu}_{3} \mathrm{Ti}_{4} \mathrm{O}_{12}$; XRD; Dielectric constant; Dielectric Loss

Abbreviations: CCTO: $\mathrm{CaCu}_{3} \mathrm{Ti}_{4} \mathrm{O}_{12}$; XRD: X-Ray Powder Diffraction

\section{Introduction}

As electronics industry is advancing day by day, need of newer materials is also increasing. With this advancement, a lot of reduction in size of electronics devices is taking place. To reduce the size of electronic devices it is necessary to reduce the size of components that are used in those devices. As Capacitor is one of the most important components of electronic devices, so lot of research is going on to reduce its size. A capacitor (originally known as condenser) is a passive two-terminal electrical component used to store energy in an electric field. The forms of practical capacitors vary widely, but all contain at least two electrical conductors separated by a dielectric (insulator), for example, one common construction consists of metal foils separated by a thin layer of insulating film. Capacitors are widely used in electrical circuits in many other electrical devices [1-3]. So this advancement is concomitant with the creation of new materials with high permittivity (called high-k dielectric materials). A higher $\mathrm{k}$ dielectric material can store more electric energy than the one with a lower value. As a result, its use in electronic devices reduces the size of the components and improves their efficiency also.

Traditional $\mathrm{BaTiO}_{3}$ based ferroelectric materials exhibit high dielectric constant. There is a problem with these materials as these have strong temperature dependence of their dielectric constants near ferro to para electric transition. $\mathrm{ACu}_{3} \mathrm{Ti}_{4} \mathrm{O}_{12}$ (where $\mathrm{A}=\mathrm{Ca}, \mathrm{Sr}, \mathrm{Ba}$ ) compounds were discovered in 1967 [1]. In recent years it has been reported that perovskite related compound $\mathrm{CaCu}_{3} \mathrm{Ti}_{4} \mathrm{O}_{12}$ (CCTO) has high dielectric constant $\left(10^{4}\right)$ and low loss tangent $\left(10^{-1}\right)[2-6]$, which is independent of temperature in the range $100-600 \mathrm{~K}$ and in the frequency range $10^{2}-10^{6} \mathrm{~Hz}$. Therefore it is suitable as thermally stable high dielectric constant material unlike compositions based on ferroelectric $\mathrm{BaTiO}_{3}$, which exhibit strong temperature dependence near their Curie temperature. Below $100 \mathrm{~K}$ its dielectric constant value drops off dramatically to around 100. Neutron powder diffraction does not show any change in crystal structure in the temperature range of 35-1273K [5-7]. 
Therefore, $\mathrm{CaCu}_{3} \mathrm{Ti}_{4} \mathrm{O}_{12}$ (ССТO) has attracted increasing scientific and technological interest because of its giant dielectric constant $\left(\varepsilon r \sim 10^{4}\right)$ with weak temperature/frequency dependence in a wide range of temperature (100-600K) and frequency. This suggests its potential application in capacitor based devices [2, 8-11].

\section{Experimental}

CCTO was prepared using conventional solid state reaction.

$$
\mathrm{CaCO}_{3}+3 \mathrm{CuO}+4 \mathrm{TiO}_{2} \mathrm{CaCu}_{3} \mathrm{Ti}_{4} \mathrm{O}_{12}+\underset{\mathrm{CO}_{2}}{\longrightarrow}
$$

In the present work, ССTO has been synthasised by solid state conventional technique. The starting powders $\mathrm{CaCO}_{3}$ (99.98\%), $\mathrm{CuO}(99.5 \%)$ and $\mathrm{TiO}_{2}$ (99.55) in stoichiometric amounts were mixed and grinded for 7-8 hours. Then it was calcined for 12 hours with intermittent grinding. After conforming the formation of monophasic compound to X-ray powder diffraction (XRD) using
$\mathrm{CuK} \alpha$ radiation. Polycrystalline powder mixed with 2\% PVA was cold pressed into pellets of diameter $15 \mathrm{~mm}$ and thickness $2 \mathrm{~mm}$ at a pressure of 7 ton in hydraulic press. Then pressed pellets were sintered at $10000 \mathrm{C}$ for 6 hours.

\section{Results and Discussion}

\section{XRD analysis}

X-ray diffraction patterns for CCTO were recorded in an X-ray diffractometer employing $\mathrm{Cu}-\mathrm{K}_{\alpha}$ radiation with a Ni filter (Figure 1). A scanning rate of $2^{0} / \mathrm{min}$ was used for recording. The formation of single phase solid solutions was confirmed by the absence characteristic lines of constituent's oxides in the XRD patterns. The XRD patterns were indexed using standard procedure. The XRD pattern obtained was compared with the available XRD pattern of CCTO using JCPDS file .The XRD pattern obtained was in accordance with the available CCTO XRD pattern (Figures $2 \& 3$ ).
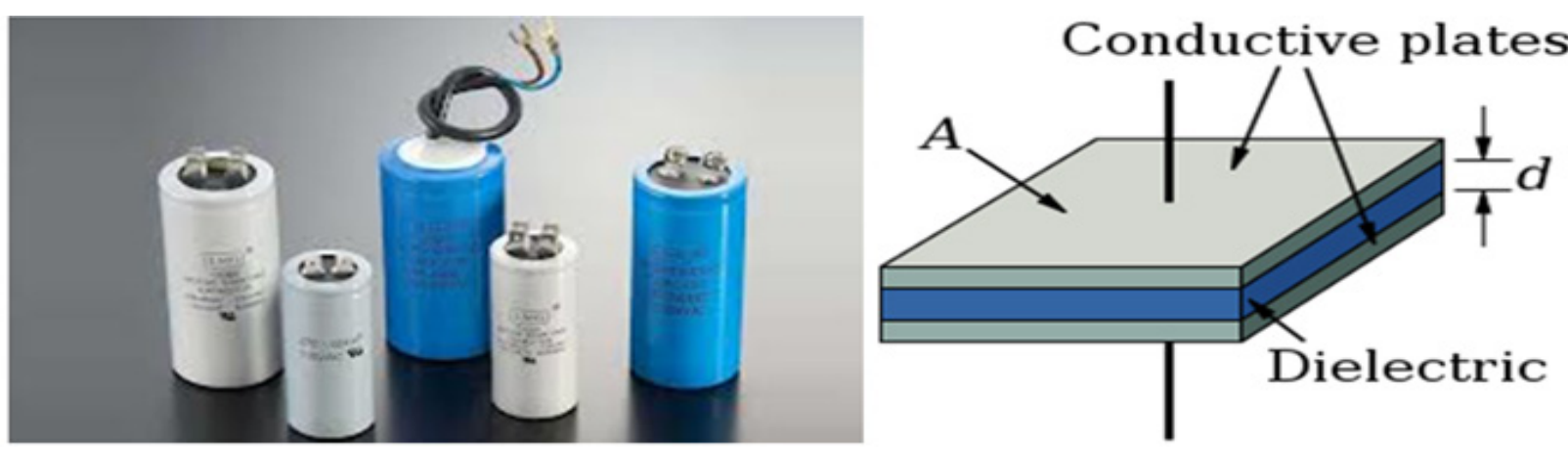

Figure 1: Capacitors and conductive plates with dielectric.

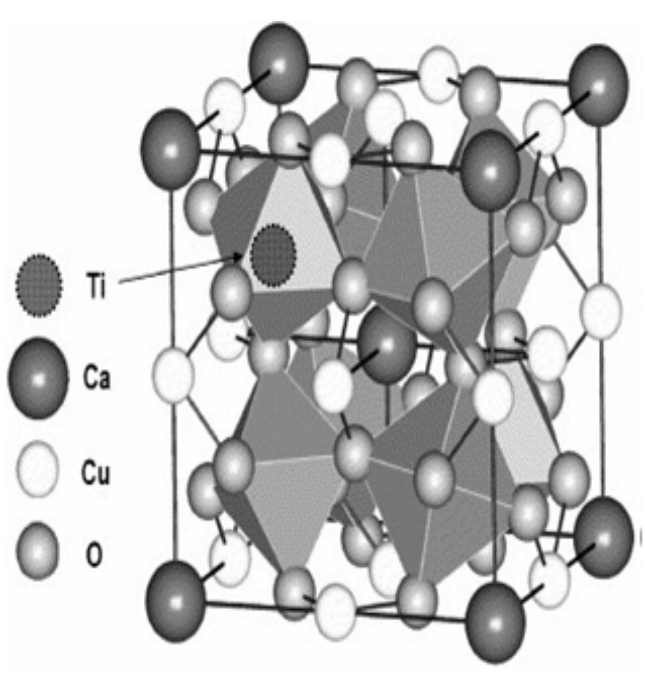

Figure 2: Crystal Structure of $\mathrm{CaCu}_{3} \mathrm{Ti}_{4} \mathrm{O}_{12}$.

\section{Dielectric Properties}

Dielectric measurement of CCTO has been performed using four probe novocontrol set up (ZG4) in a wide range of temperature starting from the room temperature. Sintered pellets were first polished by the help of different grades of emery paper and then

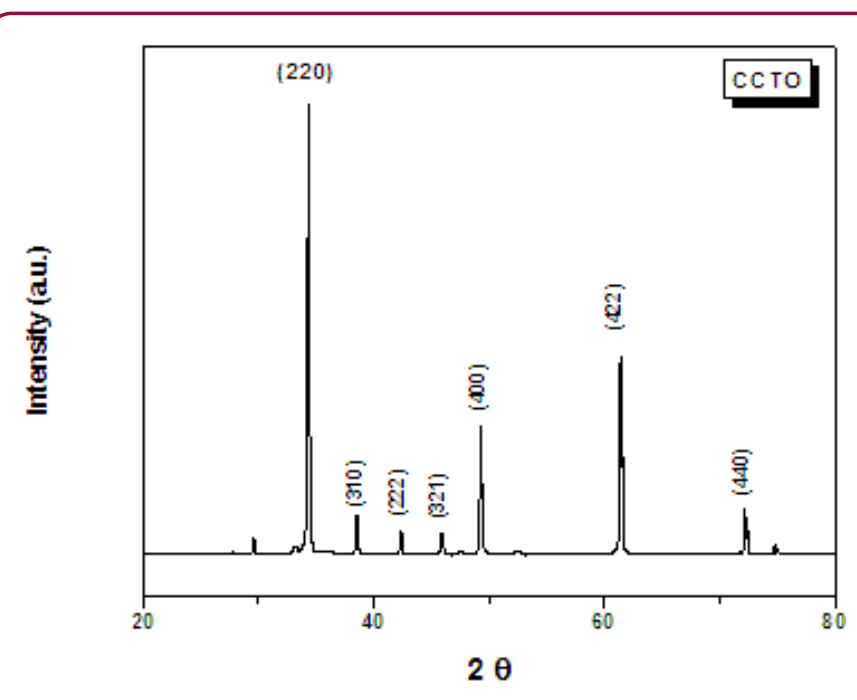

Figure 3: X-ray Diffraction Pattern of the CCTO.

silver coated which is known as electroding. Then, the sample was fixed in the sample holder. Sample has diameter of $15 \mathrm{~mm}$ and thickness $2.383 \mathrm{~mm}$ and measurement was done in the frequency range of $10^{2}$ to $2 \times 10^{7}$. Graph of frequency versus loss factor ( $\tan \delta$ ) and frequency versus $\varepsilon^{\prime}$ were recorded. 
Frequency dependence of dielectric constant of CСTO (Figure 4) at different temperature in the frequency range 102 to $2 \times 107$. The dielectric constant decreases as the frequency increases. The effective dielectric constant increases with the increase in temperature in CCTO at all the frequencies under study. At RT (room temperature) and $100 \mathrm{~Hz}$ dielectric constant value for CCTO is 5000, which increased to 9500 at $200^{\circ} \mathrm{C}$. Frequency dependence of the dielectric loss for CCTO (Figures $5 \mathrm{a} \& 5 \mathrm{~b}$ ) at different temperature in the frequency range $10^{2}$ to $2 \times 10^{7}$. Dielectric loss increases with the decrease in frequency and increase in temperature. The value of dielectric loss at RT (room temperature) and at $100 \mathrm{~Hz}$ is 0.05 , which increases to 6 at $200^{\circ} \mathrm{C}$ at same frequency (Figure 6).

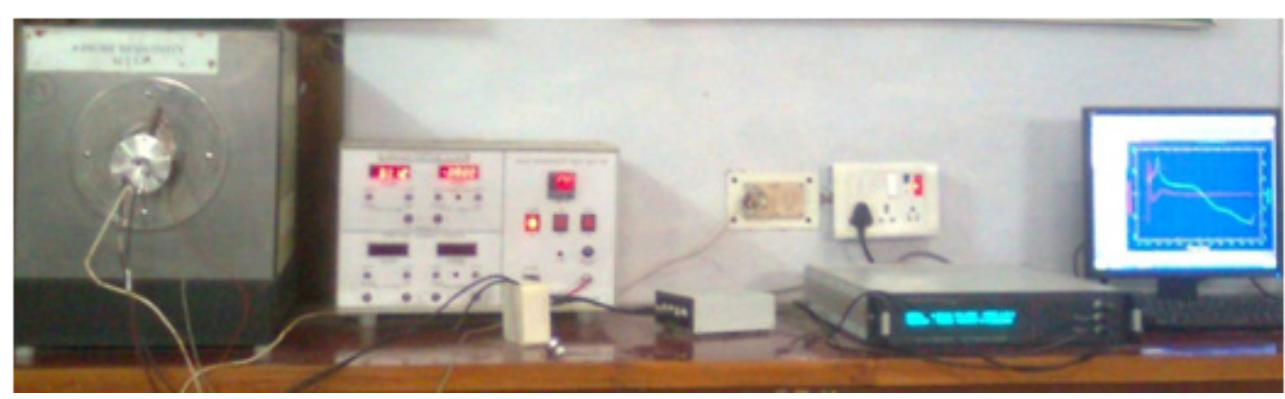

Figure 4: Novo control ZG4 Setup.

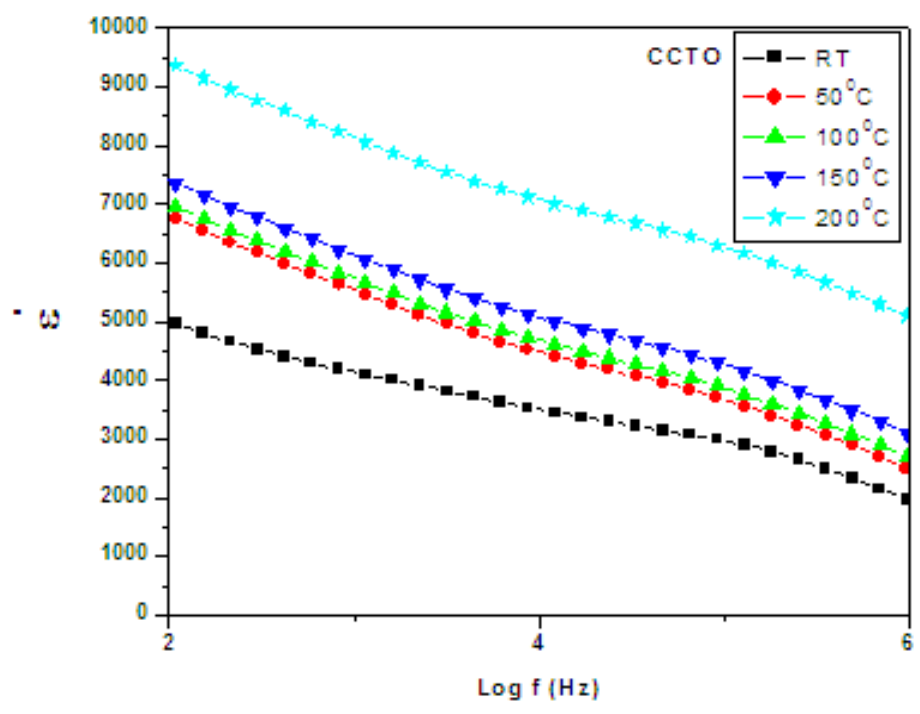

Figure 5: Novo control ZG4 Setup.
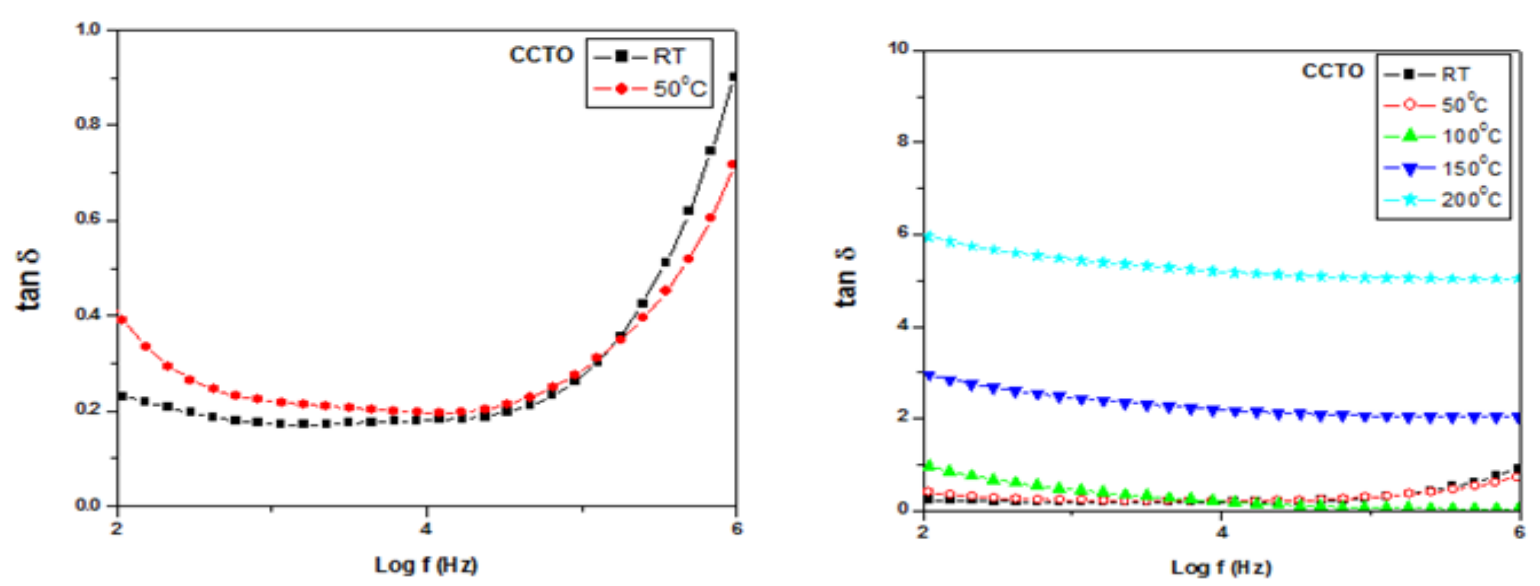

Figure 6: Novo control ZG4 Setup. 


\section{Conclusion}

The CСTO powder was successfully prepared through the conventional solid state reaction method. Single phase of CCTO was identified in calcined powders. All of the detected peaks were identified and found to be corresponding to СCTO phase. There is no additional peak detected that would indicate the formation of secondary phase. Dielectric constant increases with the increase in temperature and decreases with the increase in frequency. Dielectric loss at RT (room temperature) and at $100 \mathrm{~Hz}$ is 0.05 which is quite low. Due to high dielectric constant and low loss value it can be used for capacitor application.

\section{References}

1. Deschanvres, B Raveau, M Tollermer (1967) Bull Soc Chim Fr 11: 40774078.

2. AP Ramirez, MA Subramanian, M Gardel, G Blumberg, L Dong, et al. (2000) Solid State Commun 115: 217-220.
3. A Srivastava, KK Jana, P Maiti, D Kumar, O Parkash (2015). Journal of Engineering 2015(2015): 1-9

4. Bhattacharya SK., Tummala RR (2000), J Mater Sci, 11, 253-268.

5. S Moussa, BJ Kennedy (2001) Mater Res Bull 36: 2525-2529.

6. A Srivastava, P Maiti, D Kumar, O Parkash (2014) Composites Science and Technology 93: 83-89.

7. MA Subramanian, L Dong, N Duran, BA Resiner, AW Sleight (2000) Journal of Solid State Chem. 151: 323-325.

8. CK Chiang, KY Yin, FS Yen, CY Hwang (2001) Jl Mater Sci 36: 3809-3815.

9. A Srivastava, KK Jana, P Maiti, D Kumar, O Parkash (2015) Material Research Bulletin 70: 735-742.

10. CH Kim, KS Oh, YK Paek (2013). Journal of the Korean Ceramic Society 50(1): 87-91.

11. A Srivastava, KK Jana, P Maiti, D Kumar, O Parkash (2015). Journal of Composites70: 735-742.

\begin{tabular}{ll}
\hline $\begin{array}{l}\text { BIOMEDICAL } \\
\text { RESEARCHES }\end{array}$ & Assets of Publishing with us \\
\hline & - Global archiving of articles \\
\hline
\end{tabular}

